\title{
XML Technologies and Software Engineering
}

\author{
Cecilia Mascolo, Wolfgang Emmerich and Anthony Finkelstein \\ Department of Computer Science \\ University College London \\ Gower Street, London WC1E 6BT, UK \\ \{C.Mascolo|W.Emmerich|A.Finkelstein\}@cs.ucl.ac.uk \\ Workshop Website: http://pizza.cs.ucl.ac.uk/xse01/xse01.xml
}

The aim of the ICSE 2001 workshop on XML Technologies and Software Engineering (XSE01) is to join the efforts in the complementary areas of software engineering and XML [I]. There are two different aspects that characterize the relationship between these areas. The first addresses the use of XML and related technologies for the construction of software engineering tools and environments. The second focuses on the exploitation of XML for the development of a new generation of distributed software architectures and middleware that might provide new degrees of flexibility in terms of integration, security, and interoperability. XSE01 provides a forum to discuss both these aspects and stretches from pure research on XML and related technologies to applications and reports of industrial experience with markup languages and tools.

The intended workshop audience includes practitioners and researchers in software engineering tools, software architecture, and distributed systems. Because of the rapid evolution of XML related technologies and models, the workshop will seek to broaden the understanding and stimulate interesting collaborations. The workshop aims to identify problem areas in this domain, and discuss a research agenda to address these problems.

\section{TOPICS}

This workshop will seek to achieve several goals:

1. Identify problems and solutions in the design of software engineering tools that use XML-based interchange formats or repositories.

2. Investigate the advantages and issues arising from the use of XML in modern software architectures.

3. Establish a research community that shares results and collaborates in the development of XML based tools and strategies.
The first goal of the workshop is concerned with the investigation of issues related to the use of XML and related technologies in the software development process. XML allows the structuring of information and the developed technologies permit the access to this information in a very powerful manner. Many software development tools are taking more and more advantage of the potential of XML. Furthermore, XML leverages a long software engineering tradition of work on graph based and abstract syntax tree approaches.

The second goal of the workshop is related to the use of XML and related technologies in the development of current systems, such as middleware, web-related systems, and mobile computing applications. Structuring information in XML and the ability to exchange data in that format opens the door to the exploitation of new strategies and approaches. Research in this field is now seeing new developments in the areas of component integration, reflection, heterogeneity, and security, among others. The workshop organizers will solicit the submission of papers in these areas.

The third goal is the growth of a research community that centres around the two main areas defined above and in general around the use of XML and related technologies for the development of systems.

The programme committee seeks original high quality papers related to all aspects of use of XML technologies in software engineering context and software engineering approaches to development of XML technologies. Technologies relevant to the workshop include XML, XSL, DOM, XLink, XPath, Schema, SOAP, Biztalk, XML Query, XMI, XPL, and RDF.

Topics include, but are not limited to:

- Software Engineering with XML

- Tool inter-working

- Process definition

- Meta-Languages

- Language Engineering

- Repositories 
- Engineering Software with XML

- Development, Formalization and Analysis of new XML related technologies

- XML and Web: rendering and processing

- Use of XML in middleware development:

* integration

* security

* heterogeneity

- Meta-data

- Enterprise Application Integration

\section{Publication}

Submitted papers will be reviewed by the programme committee. Accepted papers are included in a volume of the ICSE2001 workshop proceedings, and distributed to the participant at the workshop. Accepted papers will also be made available to the general audience on the Workshop web page. For publication of the workshop proceedings, we aim to use XML technologies ourselves. The preferred submission format for papers are XML document marked-up using the DocBook DTD [2] and rendered with a DocBook stylesheet.

If a sufficient number of high-quality papers are received to warrant a more substantial publication, the organizers plan to edit a volume of Springer Verlag's LNCS and authors of accepted papers will be invited to submit a full paper.

\section{Workshop Activities}

The workshop organizers will select a few participants to give presentation to kick off discussions. Demo booth sessions will be organized, dividing the participants in 2-3 groups. At each of the sessions one of the group will give demo of their tools or approaches, while the other participants will be free to circulate and listen to the demos they are interested in. This approach allows all the participants to be able to speak about their work, and at the same time, to avoid the participants to listen to talks they are not interested in. The workshop will have challenge session (i.e., panel-like) where open issues will be discussed. We also plan a number of "capsule" tutorials (i.e., 20 minutes each) which will outline less familiar areas in an accelerated way to illuminate interesting develops and establish a common language.

\section{Workshop Committee}

\section{Chairs:}

- Cecilia Mascolo is a Lecturer in the Department of Computer Science at University College London. Her research interests are in software architecture, distributed and mobile systems. Cecilia has been involved in the application of XML and related technologies to the development of mobile code based systems, application service provision, and reflective mobile computing middleware.

- Wolfgang Emmerich is a Senior Lecturer in the Department of Computer Science at University College London. His research interests include requirements engineering and distributed objectoriented software architectures. Wolfgang is a Partner and Senior Consultant for Zuehlke Engineering (UK Ltd.). Wolfgang has consulted on several object engineering and XML projects and given several industrial training and tutorials on middleware and XML. Wolfgang is the author of a textbook on distributed objects published by John Wiley \& Sons in 2000.

- Anthony Finkelstein is a Full Professor of Software Systems Engineering at University College London, Department of Computer Science. His research is in the area of software systems engineering and in particular in requirements engineering. He has contributed to software specification methods, software development processes, tool and environment support for software development. Recent work has included significant contributions to work on specification from multiple viewpoints and to requirements traceability. His current interests are in the area of managing distributed information in software development using XML and related technologies. He is actively involved in the Software Engineering research and practitioner communities.

\section{Program Committee:}

Kenneth Anderson (U Colorado, USA)

Grady Booch (Catapulse, USA)

Paolo Ciancarini (U Bologna, Italy)

Ernst Ellmer (Zuhlke Engineering, Switzerland)

Roy Fielding (eBuilt, USA)

Harald Gall (TU Vienna, Austria)

Richard Jeliffe (Academia Sinica, Taiwan)

James Hunt (FZI, Germany)

Eve Maler (Sun, USA)

Richard Taylor (UC Irvine, USA)

Robert Tolksdorf (TU Berlin, Germany)

\section{REFERENCES}

[1] T. Bray, J. Paoli, and C. M. Sperberg-McQueen. Extensible Markup Language. Recommendation http://www.w3.org/TR/1998/REC-xml-19980210, World Wide Web Consortium, March 1998.

[2] Norman Walsh and Leonard Muellner. DocBook: The Definitive Guide. O'Reilly, 1999. 